\title{
Novel Multiphase D-shaped Fiber Optic Sensor Realized by Femtosecond Laser Machining
}

\author{
Chien-Hsing Chen ${ }^{1}$, Tzu-Ting Weng ${ }^{2}$, Jian-Neng Wang ${ }^{3}$, Chung-Wei Cheng ${ }^{4}$, Jaw-Luen Tang ${ }^{* 1}$, \\ Lai-Kwan Chau ${ }^{* 2}$ and Wei-Te $\mathrm{Wu}^{* 5}$ \\ ${ }^{* 1}$ Department of Physics, National Chung Cheng University, Minhsiung, Chiayi 621, Taiwan \\ E-mail: jawluen@ccu.edu.tw (Jaw-Luen Tang) \\ *2 Department of Chemistry and Biochemistry, National Chung Cheng University, Minhsiung, \\ Chiayi 621, Taiwan \\ E-mail: chelkc@ccu.edu.tw (Lai-Kwan Chau) \\ ${ }^{3}$ Department of Construction Engineering, National Yunlin University of Science and Technology, \\ Douliou, Yun-lin 640, Taiwan \\ ${ }^{4}$ ITRI South, Industrial Technology Research Institute, Liujia, Tainan 734, Taiwan \\ ${ }^{* 5}$ Department of Biomechatronics Engineering, National Pingtung University of Science and Tech- \\ nology, Neipu, Pingtung 912, Taiwan \\ E-mail: weite@mail.npust.edu.tw(Wei-TeWu)
}

\begin{abstract}
A novel class of multiphase D-shaped optical fiber suitable for refractive index measurements is presented. The multiphase D-shaped optical fiber was constructed by forming six D-sections in a multimode optical fiber at localized regions with femtosecond laser pulses. Each D-shaped zone covered a sensor volume of $100 \mu \mathrm{m}$ depth, $245 \mu \mathrm{m}$ width, and $1 \mathrm{~mm}$ length. Each bottom plane of a D-shaped zone forms an angle of 60 degree with the bottom plane of an adjacent D-shaped zone. The multiphase D-shaped optical fiber as a highly sensitive refractive-index sensor to detect changes in the surrounding refractive index was studied. Results show that a refractive index resolution of $2.82 \times 10^{-4}$ RIU was achieved for sucrose solutions with refractive indices in the range of 1.333 to 1.403 , suggesting that the multiphase D-shaped fibers are attractive for chemical, biological, and biochemical sensing.
\end{abstract}

DOI:10.2961/jlmn.2011.01.0017

Keywords: multiphase D-shaped fiber, femtosecond laser, sensor

\section{Introduction}

There are many types of biosensor, e.g., electrochemical biosensors, ion sensitive field effect transistors (ISFET), fiber-optic biosensors, piezoelectric quartz crystal biosensors, and others [1]. Among them, the fiber-optic biosensor has the advantages of not being affected by interference from electromagnetic noises and magnetic fields, nonionizing radiation, smaller than a conventional sensor and transfer line combined, avoiding electric shock, corrosion-proof, good biocompatibility, excellent temperature tolerance, as well as long-term stability and outstanding fatigue lifecycle. As a result, fiber optic sensors are being used extensively in various fields such as aviation, medical field, chemistry, geotechnical engineering, civil engineering and others. Based on the location where the modulation that is being sensed occurs, there are three types of fiber sensors. (1) Extrinsic Fiber Sensor: The optical fiber acts as a signal transmission line without participating in the sensing operation. After being guided to the test area, part of the light leaves the optical fiber as a result of being modulated by the external environment. The modulated light is then sent via another optical fiber to a signal generator for signal interpretation. (2) Intrinsic Fiber Sensor: The light wave basically remains in the optical fiber at all times. As it passes through the test area where the fiber jacket and cladding is stripped, the internal characteristics of the optical fiber are changed by the external environment, thus affecting certain features of the light wave (e.g., intensity, phase, polarization, and wavelength). (3) Evanescent Fiber Sensor: The loss of light energy caused by the test area is detected and is used to derivate the environmental parameters. This type of sensor is similar yet different from the extrinsic fiber sensor due to the modulation mechanism. The fiber plays a role not only as a signal transmission line but also as a sensing component.

In recent research, several optical fiber biosensors were developed combined with attenuated total reflection [2], propagating surface plasmon resonance [3] and localized plasmon resonance [4-5], respectively. Because of the protective jacket layer, the fiber can be applied in the field of communication. However, as an evanescent optical fiber sensor, the jacket layer, even the cladding layer must be stripped, and the core must be exposed to the environment. In general the plastic cladding of the optical fiber was easily stripped by $\mathrm{CO}_{2}$ laser machining to expose the fiber core [6-7]. However, the core and cladding layers of communication grade multimode optical fiber are composed of fused silica, which is a transparent, hard, brittle, and high band gap material that could not be effectively machined by long-pulsed lasers, since the peak power intensity of the long-pulsed laser is not high enough to generate a significant amount of free electrons [8-10]. Recently, femtosecond laser has been extensively used for microfabrication. The most prominent features of the femtosecond laser over 
conventional long-pulsed laser are ultra short pulse duration and very strong peak power intensity, which can photoinduce the non-linear multi-photon absorption of a material during irradiation. The material vaporizes immediately after absorbing ultra high transient pulse energy from the ultra short pulse of femtosecond laser [11-12]. It can be used to engrave on transparent, hard and brittle materials very precisely, such as optical fibers, without inducing any micro cracks and heat affected zone [13-15].

In this paper, we report a multiphase D-shaped optical fiber sensor with a direct write technique by using highpower femtosecond laser pulses. The term multiphase proposed here is used to refer to any D-shaped zone existing in the fiber with its own orientation (vector) or phase (angle) about a fixed axis perpendicular to the axial direction of the fiber. As illustrated in Figure 1, the structure of a multiphase D-shaped fiber is the one characterized by the simultaneous presence of several Dshaped zones differing in orientation or phase. In general, it is most convenient to specify a normal vector on the bottom surface of a D-shaped zone as its orientation in space. This means that the phase of a D-shaped zone is the angle between its orientation vector and the fixed axis. In our study, the orientation of the first fabricated D-shaped zone is taken as the fixed axis, thus the phase of the next fabricated D-shaped zone can be determined by rotating its bottom surface an angle with respect to the fixed axis.

In the present study we investigate a multiphase Dshaped fiber having six D-shaped zones in which the phase angle between any two adjacent $\mathrm{D}$-shaped zones is 60 degrees. Our previous studies $[14,16]$ have shown that a D-type fiber having six D-shaped zones with the same orientation (in phase) is the optimum design to be used as a highly sensitive fiber-optic localized surface plasmon resonance (FO-LPR) refractive-index sensor, suited for chemical, biological, and biochemical sensing. Furthermore, when integrated with a microfluidic chip, a six D-shaped zones FO-LPR fiber probe is also the optimum design that could provide efficient performance on biochemical binding and induce chaotic advection to enhance the microfluid mixing in the microchannel. This compact and lowcost sensor has potential applications in medical diagnostics, biochemical sensing, and environmental monitoring, and can hopefully benefit the development and application of any new and existing biosensors.

However, those previous studies are base on the design of a multi-D-shaped fiber having every D-shaped zone in the same orientation or phase, called an in-phase multiD-shaped fiber. It would be interesting to present similar studies for a multiphase D-shaped fiber and compare its performance with that of an in-phase multi-D-shaped fiber. Compared with other existing biochemical sensing schemes, such multiphase D-shaped fiber sensors seem more promising and favorable. The physical and optical properties of multiphase D-shaped fibers for biosensor applications have yet to be fully exploited, which may be achieved by a detailed study of the effects of phase arrangement and dimensions (length, width, and depth). Furthermore, when modified with gold nanoparticles and integrated with a microfluidic chip, the multiphase D-shaped optical fiber as a FOLPR sensor is expected to have better performance on biochemical binding, micro-fluid mixing, and sensitivity of chemical sensing than that of the in-phase multi-D-shaped fiber.

A communication grade multimode optical fiber (Corning 62.5/125 optical fiber) was adopted in the present study. The optical fiber was composed of a silica-based core $(62.5 \mu \mathrm{m}$ in diameter), and cladding and polymeric jacket with outer diameters of $125 \mu \mathrm{m}$ and $250 \mu \mathrm{m}$, respectively. Figure 1 is an illustration diagram of the fabricated multiphase D-shaped optical fiber. There are six D-shaped zones with each bottom plane of a D-shaped zone forms an angle of 60 degree with the bottom plane of an adjacent D-shaped zone. For a single D-shaped zone, the depth is $100 \mu \mathrm{m}$ measured from the surface of the polymer jacket layer, and the length is $1 \mathrm{~mm}$. The distance between two neighboring D-shaped zones is $1 \mathrm{~mm}$. When a Dshaped fiber is microstructured to this depth, the exposed surface of the fiber can be modified with gold nanoparticles, where most of the nanoparticles are uniformly distributed and immobilized on the surface $[13,15]$.

The operating principle of sensing is based on attenuated total internal reflection (ATR) via multiple internal reflections along the fiber and the attenuated light intensity of the multiphase D-shaped fiber changes linearly with an increase of the surrounding refractive index. The loss of light energy caused by the sensing portion of the fiber is detected by a sensor interrogation system. In this scheme the fiber plays a role not only as a signal transmission line but also as a sensing component. The ability of the multiphase D-shaped fiber as a highly sensitive refractive-index sensor to detect changes in the surrounding refractive index was also studied.
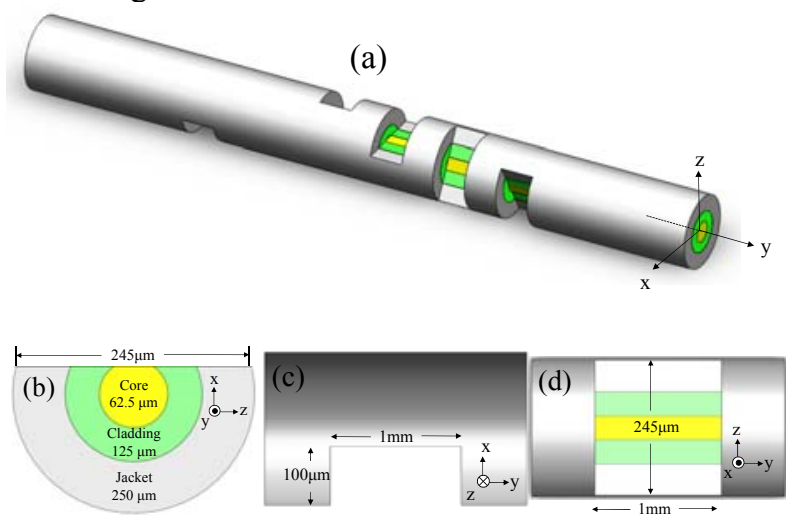

Fig. 1 Various diagrams illustrating a multiphase D-shaped optical fiber: (a) perspective view; (b) cross-sectional view at $\mathrm{x}-\mathrm{Z}$ plane; (c) cross-sectional view at x-y plane;

(d) cross-sectional view at z-y plane.

\section{Sensor Fabrication}

A femtosecond laser micromachining system, as illustrated in Figure 2, was used for engraving the trench on the optical fiber. The femtosecond laser was a regenerative amplified mode-locked Ti: sapphire laser with pulse duration of $\sim 120$ fs after the compressor, peak wavelength at $800 \mathrm{~nm}$, repetition rate of $1 \mathrm{kHz}$, and maximum pulse energy of $\sim 3.5 \mathrm{~mJ}$. The energy of the linear polarized Gaussian laser beam was adjusted by a rotatable half-wave plate and a polarizing beam splitter (PBS). A certain fraction of the laser beam was split off by a beam splitter (BS) and the 
laser energy was measured by a power detector. The number of laser shots applied to the sample was controlled by an electromechanical shutter. The laser beam was tightly focused onto the fiber by a 10x objective lens (numerical aperture 0.26, M Plan Apo NIR, Mitutoyo) mounted on a Z stage. The raw multimode optical fiber was fastened by the fiber clamping apparatus as shown in Figure 3. The clamping apparatus was made of polymethylmethacrylate (PMMA), and it contained four parts: two rectangular slabs and two hexagonal slabs. There was a channel for placing the optical fiber and six rectangular hollow zones as fabrication zones in the rectangular slabs. The function of the hexagonal slabs was to fix the rectangular slabs with raw multimode optical fiber, and they were turned after finishing a D-shaped zone in order to provide an angle between the bottom planes of two adjacent D-shaped zones. The fiber was mounted on the fiber clamping apparatus with its longitudinal axis perpendicular to the beam path of the laser pulses. The multiphase D-shaped trench under fabrication was translated by a computer controlled X-Y micropositioning stage with error less than $1 \mu \mathrm{m}$. The fabrication process was monitored in situ by a charge-coupled device (CCD).

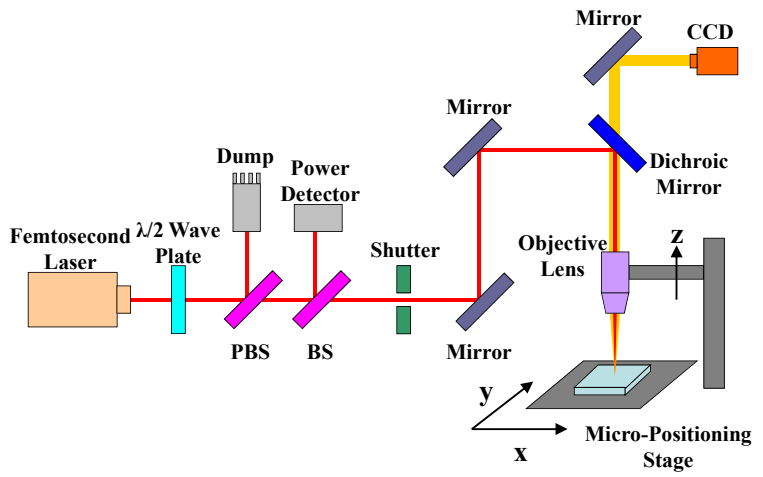

Fig. 2 Experimental setup of a femtosecond laser micromachining system.

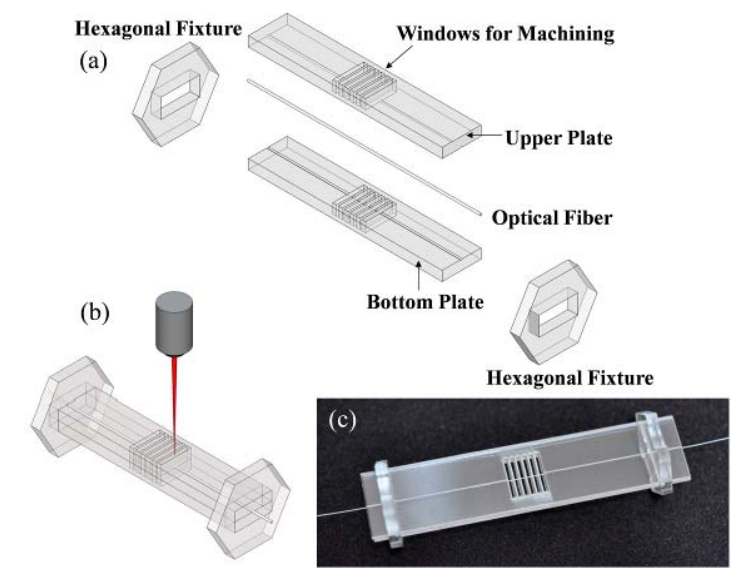

Fig. 3 The fiber claming apparatus for fastening the optical fiber. (a) Perspective view showing the assembly drawing of the optical fiber clamp. (b) Illustration of a fiber mounted on the optical fiber clamp in which the fiber is illuminated by a focused laser beam.

(c) Photograph of the optical fiber clamp.

Figure 1 and Figure 5 show the representative diagrams and SEM/AFM images for the fabricated multiphase D- shaped optical fibers, respectively. The diagram shows that the depth of a single D-shaped zone was $100 \mu \mathrm{m}$ and the length was $1 \mathrm{~mm}$. The space between the core center and the flat surface of the fiber was $25 \mu \mathrm{m}$. It indicates that the core of the fiber has been exposed and part of the jacket layer has provided enough mechanical strength for further processing. Since the material properties of the jacket layer and cladding layer are much different, there are three main steps used for fabricating the multiphase D-shaped optical fibers (vide infra).

As illustrated in Figure 1 and Figure 4, we assign the longitudinal (or axial) axis of the optical fiber with y axis, the cross-sectional direction of the optical fiber with $\mathrm{x}$ axis, and the direction parallel to the beam path of laser pulses, with $\mathrm{z}$ axis. The processing parameters used for the fabrication of a multiphase D-shaped fiber include: laser power, the height of the laser focus spot (focus height), laser focus spot size, scanning width, scanning length, scanning pitch and scanning speed. To obtain a higher resolution of microstructure within optical fibers, the pulse energy was carefully adjusted such that only the peak region of the Gaussian beam distribution is above ablation threshold, resulting in a small focus spot size of $\sim 5 \mu \mathrm{m}$. The scanning pitch, distance between two consecutive laser shots, was tuned to half of the focus spot size of $\sim 2.5 \mu \mathrm{m}$ in order to achieve an efficient micromachining with minimal heat diffusion. When processing with the laser pulses, the scanning width and length determine a defined surface of region for removal of the fiber materials in $\mathrm{x}$ and $\mathrm{y}$ directions, respectively. Based on the way we used to machining the optical fiber (vide infra), this results in a 2-D section in the D-shaped zone (see Figure 1 and Figure 4) for which its dimensions of depth and width are given by the scanning width and length, respectively.

The focus height is defined as the distance between the focus spot and the surface of polymer jacket in optical fiber. During the course of laser machining with three major steps, the focus height was varied at different steps and the other parameters were constantly set. Typical operating parameters are $20 \mathrm{~mW}$ average power, $5 \mu \mathrm{m}$ focus spot size, $2.5 \mu \mathrm{m}$ scanning pitch, $100 \mu \mathrm{m}$ scanning width, 1 $\mathrm{mm}$ scanning length, and $0.1 \mathrm{~mm} / \mathrm{s}$ scanning speed. The focus height was set to $0 \mu \mathrm{m}, 60 \mu \mathrm{m}$, and $120 \mu \mathrm{m}$ for steps 1,2 , and 3 , respectively. To rapid formation of a single Dshaped fiber, a raster scan technique, similar to that of reconstruction of an image in television by sweeping the screen in a line-by-line manner, was implemented. In a raster scan, it scans the whole working area of the fiber sample, generally from left to right horizontally at a steady rate, while progressing vertically from top to down of the fiber sample.

The three main steps to form a multiphase D-shaped fiber are described as follows. In Step 1, turning off the laser power, a reference position at the surface of polymer jacket (the blue dot point in Figure 4 (a)) or the origin of the coordinate system was located by moving the fiber sample to this point using the micro-positioning stage. The fiber sample was then moved to $\mathrm{x}=25 \mu \mathrm{m}$ (the red dot point in Figure 4 (a)) as a starting point of the machining process. The focus height was adjusted to the surface of the polymer jacket, $\mathrm{z}=0$, and the following processing parameters were chosen: $20 \mathrm{~mW}$ laser average power, $5 \mu \mathrm{m}$ 
focus spot size, $2.5 \mu \mathrm{m}$ scanning pitch, $0.1 \mathrm{~mm} / \mathrm{s}$ scanning speed, $1.0 \mathrm{~mm}$ scanning length, and $100 \mu \mathrm{m}$ scanning width. Turning on the power of laser source, a raster scan was performed by sweeping the defined area in the fiber sample from left to right along the y axis with the scanning speed, while advancing from top to bottom along the $\mathrm{x}$ axis with a step size of scanning pitch. As a result, the polymer jackets of the fiber sample were partially removed as shown in Figure 4 (b). After the completion of Step 1, partial jackets of the fiber sample were exposed.

To proceed on the next step, the laser was turned off and the fiber sample was back to the starting point. In Step 2 , the focus height was varied and tuned to $\mathrm{z}=-60 \mu \mathrm{m}$ (shown in Figure 4 (c)), a position inside the polymer jacket of the fiber. Using the same processing parameters as Step 1 and turning on the laser source, the raster scan on the defined area of the fiber sample was performed so that the remaining polymer jackets and partial claddings of the fiber sample were removed as illustrated in Figure 4 (c). To complete the final step, the fiber was back to the starting position and the focus height was adjusted to $\mathrm{z}=-120 \mu \mathrm{m}$, a position inside the core of the fiber. In Step 3, the raster scan was performed again with the same processing parameters as in Step 1, resulting in the removal of the remaining claddings and partial core materials of the fiber, as shown in Figure 4 (d). As a result, a single D-shaped zone with dimensions of $100 \mu \mathrm{m}$ depth, $245 \mu \mathrm{m}$ width, and $1 \mathrm{~mm}$ length was fabricated after these three main steps. To fabricate the next D-shaped zone, the fiber clamping apparatus was rotating by an angle of 60 degrees, and the fiber sample was translated to the next rectangular hollow window of the fiber clamping apparatus. A new D-shaped zone was then fabricated by repeating the above three main steps. These processes were repeated until all the six Dshaped zones have been made.

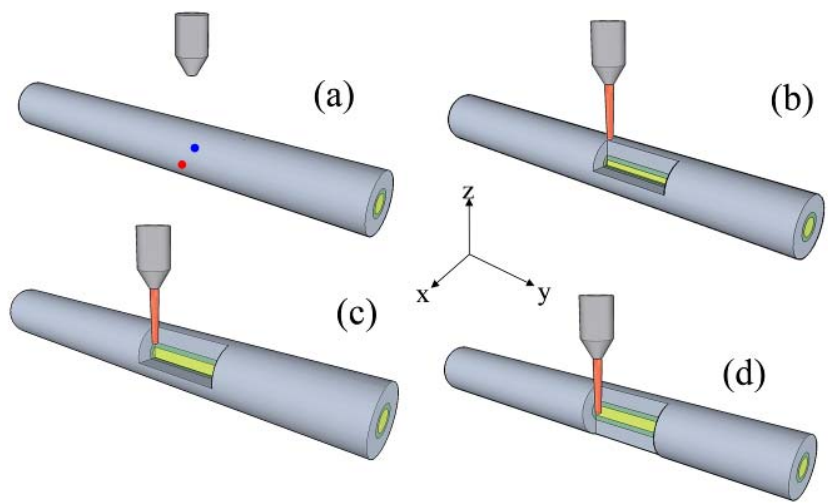

Fig. 4 Schematic diagrams of the three main steps for making a D-shaped zone by the femtosecond laser. (a) The initial setup of the starting point for the machining process; (b) The first step for removing the partial polymer jacket layer; (c) The second step for removing the remaining jackets and partial cladding layers;

(d) The final setup of finishing a D-shaped zone by removing the remaining claddings and partial core materials.
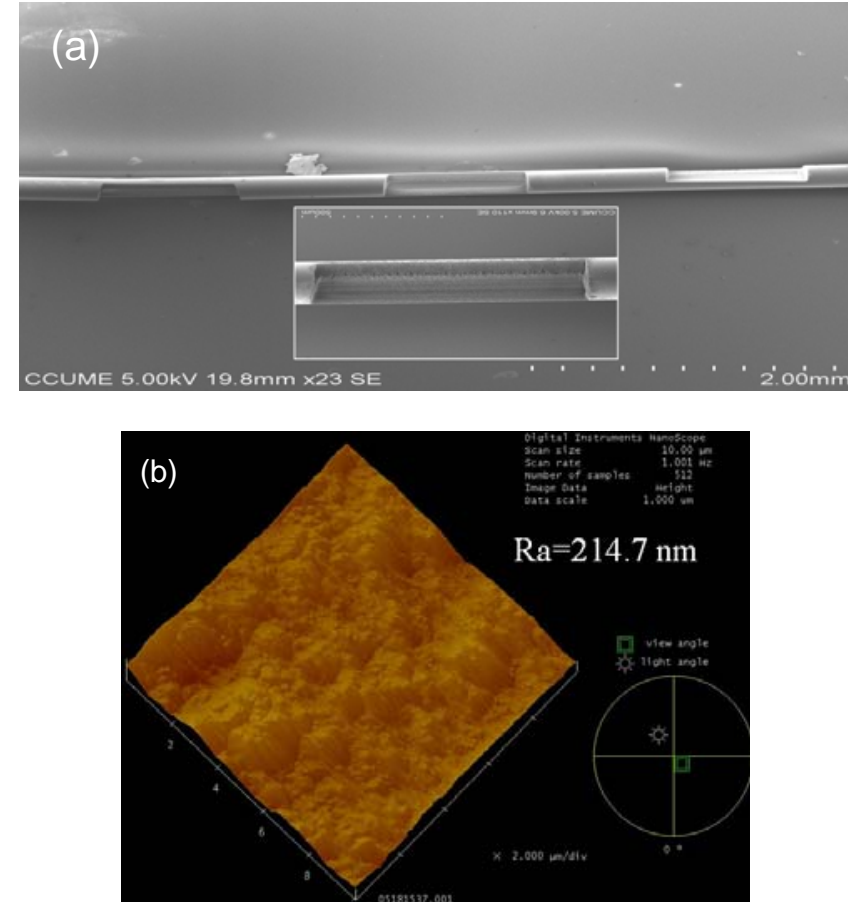

Fig. 5 (a) SEM image of a multiphase D-shaped optical fiber with an enlarged image in the inset. (b) AFM image showing the surface mean roughness.

\section{Refractive Index Measurements}

Figure 6 is the experimental setup for refractive index sensing measurements with the multiphase D-shaped optical fiber sensor. The fiber-optic sensing system used to measure the transmission power of the sensor was consisted of a function generator (model GFG-8255A, Good Will Instrument, Inc.), a light-emitting diode (LED) light source (model EHP-AX08LS-HA/SUG01-P01, Everlight Electronics Co., Ltd), a multiphase D-shaped fiber sensor, a microfluidic chip, a photodiode (model 2001, New Focus, Inc.), a lock-in amplifier (model 7225R, EG\&G Instrument, Inc.) and a computer for data acquibitionterrogation of the sensor signal based on intensity modulation was performed by launching a LED light source propagating through the multiphase D-shaped fiber sensor and then to a photodiode. The LED as an excitation light source was modulated by a function generator with a square wave current at a frequency of $1 \mathrm{kHz}$ and a voltage of $3.5 \mathrm{~V}$. Through a fiber collimator (model F240FC-A, Thorlab), light with a wavelength of $530 \mathrm{~nm}$ emitted from the LED was coupled into the optical fiber and was carried through the sensing portion of the D-shaped fiber which was immersed in a sucrose solution of various concentrations. For measurements of transmission power, the ATR signals emitted from the sensing fiber was measured by a photodiode and the light signal was converted into electronic signal in voltage. A lock-in amplifier operating at 1 $\mathrm{kHz}$ chopping frequency was used for phase-shift detection of the photodiode output signal and for increasing the signal to noise ratio. With the lock-in, low level optical signals can be detected with full-scale voltage sensitivities down to $2 \mathrm{nV}$ and dynamic reserve exceeding $100 \mathrm{~dB}$. The output from the lock-in-amplifier was recorded at a sampling rate of $1 \mathrm{~Hz}$ using a computer with a data acquisition system. For each run of transmission power measurement, the aver- 
age transmission power can thus be obtained by evaluating a series of 500 data samples. In this scheme the computer associated with the photodiode and the lock-in amplifier carried out the light intensity demodulation and signal processing for obtaining the output signal of the sensor.

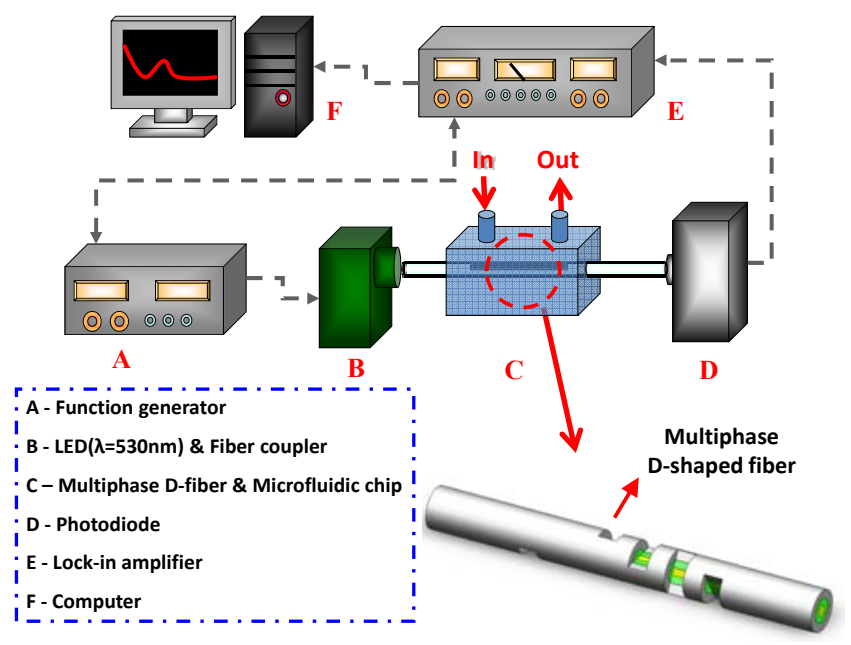

Fig. 6 Schematic of the experimental setup for refractive index measurements with the multiphase D-shaped optical fiber. The inlet and outlet ports of the microfluidic chip are used to infuse sucrose solution flowing through the sensing fiber.

The ability of the multiphase D-shaped optical fibers to detect changes in the surrounding refractive index was studied. The surrounding refractive index was adjusted by using sucrose solutions with various concentrations [17]. The relationship between refractive index and concentration of sucrose solution in the range of 1.333 to 1.403 is shown in Figure 7. The transmitted ATR signal of the multiphase D-shaped fiber excited by the LED light source changed linearly with an increase of the surrounding refractive index. Sucrose solutions of various concentrations were used to measure the sensitivity of the multiphase D-shaped fiber sensor. The fiber sensor was immersed in a sucrose solution and its transmission power or intensity was measured with the photodiode and lock-inamplifier. Transmission power or intensity was used as the sensor response in this study, instead of converting transmission power to transmittance or absorbance. The performance of the sensor was evaluated by analyzing the plot of transmission power or intensity versus refractive index. For precise refractive index measurement, we kept the experimental setup and sample solution at a constant ambient temperature (within $0.1{ }^{\circ} \mathrm{C}$ fluctuation). The sensing fiber was placed inside the microfluidic chip and a small fixed magnitude of tension was applied to minimize bending of the fiber. Therefore, the results reported here were not influenced by temperature, strain and bending effects.

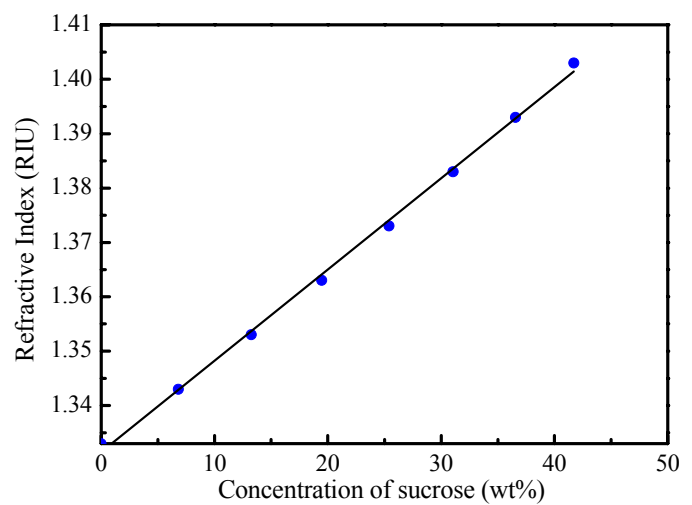

Fig. 7 Refractive index of the sucrose solution at different concentrations.

When the concentration and, hence, the refractive index of a sucrose solution increased in the range of 1.333-1.403, the transmitted light intensity of the fiber sensor exhibited a linear increase in the output power. Figure 8 shows the time course of sensor response as sucrose solutions of increasing concentration was injected into the sensing microfluidic chip. After the injection of the final sucrose solution, the sensing microfluidic chip was injected with water. It can be seen that the sucrose solution could be eluted completely and quickly, and the sensor response was back to the original intensity level. The sensor response was reversible because such injection and elution processes were repeatable.

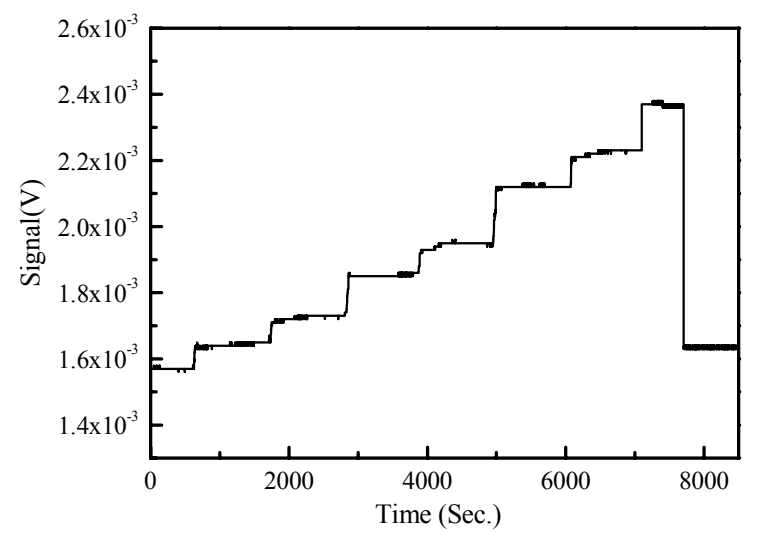

Fig. 8 Plot of temporal response of the fiber sensor with respect to injection of sucrose solutions of increasing concentrations or refractive indices.

A linear regression method was employed to analyze the relationship between sensor response and refractive index changes. This method calculated the best-fitting linear equation for the observed data using the least squares approach. The slope of the linear fit to the measured sensor response at various surrounding refractive index changes was determined as the refractive index sensitivity of the investigated fiber sensor. Figure 9 shows a linear fit (correlation coefficient $R=0.9901$ ) of the plot of sensor response as a function of the refractive index. Results for a sensing fiber with multiphase D-shaped zones show that the refractive index sensitivity of the fiber sensor is $1.17 \times 10^{-2}$ $\mathrm{V} /$ refractive index unit (RIU), which leads to a refractive index resolution (sensor resolution $=3 \sigma / \mathrm{m}, \sigma=$ standard 
deviation of sensor response in measuring the blank sample, $m=$ slope) of $2.82 \times 10^{-4}$ RIU over refractive indix range of 1.333 to 1.403 .

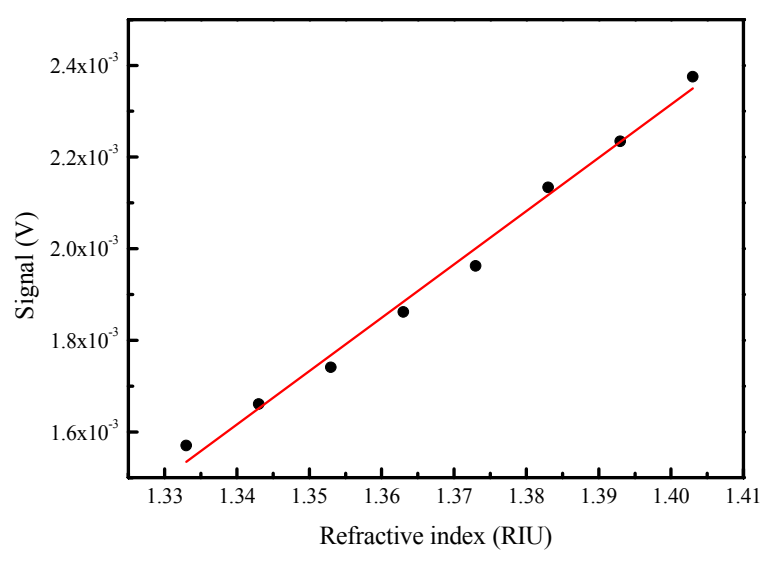

Fig. 9 Plot of sensor response versus refractive index of the sucrose solution.

Compared with other existing refractive index sensing schemes [13-15, 18-19], such a sensor performance is promising and favorable. The previous study [14] of a fiber sensor with in phase six D-shaped zones has reported a resolution of $3.24 \times 10^{-4}$ RIU, which is similar to the present multiphase D-shaped fiber, having a resolution of $2.82 \times 10^{-4}$ RIU. In these two studies, sensing scheme, sensing length, width and depth of D-shaped zone, and number of D-shaped zones are the same except the phase arrangements of D-shaped zones. As mentioned earlier, a FO-LPR sensor associated with multiphase D-shaped fiber is expected to have better performance on biochemical binding, micro-fluid mixing, and sensitivity for chemical sensing than that of the in-phase multi-D-shaped fiber. Our fiber sensor is low-cost and compact, has comparable or better resolution, and can be used remotely. The results reported here demonstrate that the multiphase D-shaped fibers are attractive for chemical, biological, and biochemical sensing.

\section{Conclusions}

Studies presented here successfully demonstrate the feasibility of fabricating a class of highly sensitive refractive-index sensor based on the multiphase D-shaped optical fiber written by femtosecond laser pulses. The multiphase D-shaped fibers were fabricated using multimode standard communications step-index optical fiber with a core diameter of $62.5 \mu \mathrm{m}$ and with an outer cladding diameter of 125 $\mu \mathrm{m}$. The realization of the sensor is through the measurement of transmitted light intensity of the sensing fiber. When exposing the D-shaped fiber sensor to sucrose solutions of increasing refractive index, the sensor response increased linearly. By transmission power interrogation, we demonstrated that the multiphase D-shaped fiber sensors can provide a refractive index resolution of $2.82 \times 10^{-4}$ RIU over the refractive index range of 1.333 to 1.403 . Such a high sensitivity fiber-optic refractive index sensor is suitable as a chemical or biological sensor. The advantages of this type of sensor are relatively simple of construction, compact, low cost, and ease of use. Moreover, the sensor has the potential capability for on-site, in vivo, and remote sensing, and has the potential of being disposable sensors.

\section{Acknowledgments}

Support of this research by ITRI South (Taiwan, R.O.C.) through Grant No. B200-97G270001884, the National Science Council (Taiwan, R.O.C.) through Grant No. NSC 95-3114-P-194-001-MY3, NSC 96-2112-M-194-004-MY3, NSC 99-2120-M-194-004-CC1, NSC 99-2221-E-224-055MY2, and NSC 99-2112-M-194-004-MY3 are gratefully acknowledged.

\section{References}

[1] J. Janata: "Principles of Chemical Sensors," (Plenum Press, New York and London, 1989).

[2] K. Welford: IOP Short Meeting Series No.9, Inst. Phys., (1987) 25.

[3] D.S. Wang and M. Kerker: Phys. Rev. B, 24, (1981) 1777.

[4] L. K. Chau, Y. F. Lin, S. F. Cheng, and T. J. Lin: Sensors \& Actuators B Chem., 113, (2006) 100.

[5] E. Hutter and J. H. Fendler: Adv. Mater., 16, (2004) 1685.

[6] S. F. Cheng and L. K. Chau: Anal. Chem., 75, (2003) 16.

[7] J. L. Tang, S. F. Cheng, W. T. Hsu, T. Y. Chiang, and L. K. Chau: Sensors \& Actuators: B. Chemical, 119, (2006) 105.

[8] B. C. Stuart, M. D. Feit, S. Herman, A. M. Rubenchik, B. W. Shore, and M. D. Perry: J. Opt. Soc. Am. B, 13, (1996) 459.

[9] W. Kautek, Kriiger, J. M. Lenzner, S. Sartania, C. Spielmann, and F. Krausz: Appl. Phys. Lett., 69, (1996) 3146.

[10]D. Ashkenasi, H. Varel, A. Rosenfeld, S. Henz, J. Herrmann, and E. E. B. Campbell: Appl. Phys. Lett., 72, (1998) 1442.

[11]A. Ben-Yakar and R. L. Byer: J. Appl. Physi., 96, (2004) 5316.

[12]R. Gattass and E. Mazur: Nature Photonics, 2, (2008) 219.

[13] C. H. Chen, T. C. Chao, W. Y. Li, W. C. Shen, C. W. Cheng, J. L. Tang, L. K. Chau, and W. T. Wu: Journal of Laser Micro/Nanoengineering, 5, (2010) 1.

[14] C. H. Chen, T. C. Chao, J. L. Tang, and W. T. Wu: Sensors, 10, (2010) 4794.

[15] C. H. Chen, T. C. Tsao, W. Y. Li, W. C. Shen, C. W. Cheng, J. L. Tang, C. P. Jen, L. K. Chau, and W. T. Wu: Microsystem Technologies, 16, (2010) 1207.

[16] C. T. Huang, C. P. Jen, T. C. Chao, W. T. Wu, W. Y. Li, and L. K. Chau: Sensors, 9, (2009) 6456.

[17] T. C. Hwang: "Experiments in Physical Chemistry," (in Traditional Chinese, Gaulih Book Co., Taipei, 1994).

[18] J. L. Tang and J. N. Wang: Sensors, 8, (2008) 171.

[19] J. Rheims, J Köser, and T Wriedt: Meas. Sci. Technol., $8,(1997) 601$

(Received: June 07, 2010, Accepted: February 22, 2011) 